\title{
THE DISTRIBUTION OF PICARD DIMENSIONS
}

\author{
Dedicated to Professor Mitsuru Ozawa on the occasion of his 60th birthday
}

\section{By Mitsuru Nakai and Toshimasa Tada}

The purpose of this paper is to show that Picard dimensions of densities on the punctured unit disk cover all countable cardinal numbers as well as the cardinal number of continuum.

Before stating our result more precisely we first fix terminologies. We denote by $Q$ the unit punctured disk $0<|z|<1$ which is viewed as an end of the punctured sphere $0<|z| \leqq \infty$ so that the unit circle $|z|=1$ is the relative boundary $\partial \Omega$ of $\Omega$ and the origin $z=0$ is the ideal boundary $\delta \Omega$ of $\Omega$. By a density $P$ on $\Omega$ we mean a nonnegative locally Hölder continuous function $P(z)$ on $\bar{\Omega}=\Omega \cup \partial \Omega$ so that $P$ may or may not have singularity at $z=0$. With a density $P$ on $\Omega$ we associate the class $P P(\Omega ; \partial \Omega)$ of nonnegative continuous functions $u$ on $\bar{\Omega}$ such that $u$ satisfies the following elliptic equation

$$
4 \frac{\partial^{2}}{\partial z \partial \bar{z}} u(z)=P(z) u(z)
$$

on $\Omega$ and vanishes on $\partial \Omega$. We also denote by $P P_{1}(\Omega ; \partial \Omega)$ the subclass of $P P(\Omega ; \partial \Omega)$ consisting of functions $u$ with the following normalization

$$
-\int_{\partial \Omega} \frac{\partial}{\partial|z|} u(z)|d z|=2 \pi \text {. }
$$

The Choquet theorem (cf. e.g. Phelps [5]) yields that there exists a bijective correspondence $u \longleftrightarrow \mu$ between $\operatorname{PP}_{1}(\Omega ; \partial \Omega)$ and the set of probability measures $\mu$ on the set ex. $P P_{1}(\Omega ; \partial \Omega)$ of extremal points of the convex set $P P_{1}(\Omega ; \partial \Omega)$ such that

$$
u=\int_{\mathrm{ex} . P P_{1}(\Omega ; \partial \Omega)} v d \mu(v) .
$$

Thus the set ex. $P P_{1}(\Omega ; \partial \Omega)$ is essential for the class $P P_{1}(\Omega ; \partial \Omega)$, and the cardinal number $\#\left(\operatorname{ex} . P P_{1}(\Omega ; \partial \Omega)\right)$ of the set ex. $P P_{1}(\Omega ; \partial \Omega)$ is referred to as the Picard dimension of a density $P$ at the ideal boundary $\delta \Omega$ of $\Omega, \operatorname{dim} P$ in notation, i.e.

The main result of this paper was announced in [4]. To complete the present work both of the authors were supported in part by Grant-in-Aid for Scientific Research, No. 434005, Japanese Ministry of Education, Science and Culture.

Received February 8, 1983 


$$
\operatorname{dim} P=\#\left(\operatorname{ex} . P P_{1}(\Omega ; \partial \Omega)\right) .
$$

We are interested in the range $\operatorname{dim} \mathscr{D}$ of the mapping $P \rightarrow \operatorname{dim} P$ from the totality $\mathscr{D}$ of densities $P$ on $\Omega$ to the set of cardinal numbers. It is easily seen (cf. e.g. [3]) that $\operatorname{dim} P \geqq 1$ for any density $P$ on $\Omega$. Since $P P_{1}(\Omega ; \partial \Omega)$ is a subset of the space $C(\Omega)$ of real valued continuous functions on $\Omega$ which is separable, the well known fact $\sharp(C(\Omega))=\mathrm{c}$ implies that $\operatorname{dim} P \leqq c$, where $\mathrm{c}$ is the cardinal number of continuum. Therefore we have

$$
1 \leqq \operatorname{dim} P \leqq c \quad \text { (i.e. } \operatorname{dim} \mathscr{D C}[1, \mathrm{c}])
$$

for every density $P$ on $\Omega$, where $[1, c]$ is the interval consisting of cardinal numbers $\mathfrak{m}$ such that $1 \leqq \mathfrak{m} \leqq c$. We denote by $N$ the set of positive integers and by $\mathfrak{a}$ the countably infinite cardinal number $\# N$ and set $\boldsymbol{E}=\boldsymbol{N} \cup\{\mathfrak{a}, \mathfrak{c}\}$. The primary purpose of this paper is to prove the following result:

The MaIn Theorem. There exists a density $P_{\mathrm{m}}$ on $\Omega$ for any cardinal number $\mathfrak{m}$ in $\Xi$ such that $\operatorname{dim} P_{\mathfrak{m}}=\mathfrak{m}$.

Therefore we have $\Xi \subset \operatorname{dim} \mathscr{D C}[1, \mathfrak{c}]$ so that $\operatorname{dim} \mathscr{D}=\Xi$ if we assume the continuum hypothesis $\Xi=[1, \mathrm{c}]$. The proof is divided into two parts : the existence of canonically associated densities discussed in nos. 1-6, and three examples of relative harmonic dimensions considered in nos. 8-14. The deduction of the main theorem from the above two parts is given in no. 7 .

\section{$\S 1$. Canonically associated densities.}

1. A sequence $\left\{K_{n}\right\}_{1}^{\infty}$ of continua $K_{n}$ possibly empty in $\Omega$ will be referred to as a $\mathcal{K}$-sequence in $\Omega$ if $K_{n} \cap K_{m}=\varnothing(n \neq m), W=\Omega-\bigcup_{1}^{\infty} K_{n}$ is connected, and $\left\{K_{n}\right\}$ converges to $\delta \Omega: z=0$, i.e. there exist only a finite number of $K_{n}$ such that $K_{n} \cap\{\varepsilon \leqq|z|<1\} \neq \varnothing$ for every $\varepsilon>0$. We denote by $\mathcal{K}(\Omega)$ the set of $\mathcal{K}$ sequences in $\Omega$. The relative boundary $\partial W$ of the region $W=\Omega-\bigcup_{1}^{\infty} K_{n}$ for a $\mathcal{K}$-sequence $\left\{K_{n}\right\}_{1}^{\infty}$ is $\partial W=(\partial \Omega) \cup\left(\cup_{1}^{\infty} \partial K_{n}\right)$. We then consider the class $H P(W ; \partial W)$ of nonnegative harmonic functions on $W$ with vanishing boundary values on $\partial W$ and the subclass $H P_{1}(W ; \partial W)$ of $H P(W ; \partial W)$ consisting of those functions $u$ with the normalization (2). Similarly to the Picard dimension we define the relative harmonic dimension, $\operatorname{dim}\left\{K_{n}\right\}$ in notation, of a $\mathcal{K}$-sequence $\left\{K_{n}\right\}$ at $\delta \Omega: z=0$ by

$$
\operatorname{dim}\left\{K_{n}\right\}=\#\left(\operatorname{ex} . H P_{1}(W ; \partial W)\right) .
$$

It is easy to see that, as in the case of Picard dimensions, $1 \leqq \operatorname{dim}\left\{K_{n}\right\} \leqq c$ for any $\mathcal{K}$-sequence $\left\{K_{n}\right\}$ in $\Omega$. We will see in $\S 2$ that the range $\operatorname{dim} \mathcal{K}(\Omega)$ of the mapping $\operatorname{dim}: \mathcal{K}(\Omega) \rightarrow$ cardinal numbers $\}$ also contains $\Xi$.

2. Suppose that each continuum $\bar{Y}_{n}$ in a $\mathcal{K}$-sequence $\left\{\bar{Y}_{n}\right\}$ in $\Omega$ is the closure of a Jordan region $Y_{n}$ in $\Omega(n=1,2, \cdots)$. Such a $\mathcal{K}$-sequence will be 
referred to as a $\gamma$-sequence in $\Omega$ and we denote by $q(\Omega)$ the class of $a$-sequences in $\Omega$ so that $y(\Omega) \subset \mathcal{K}(\Omega)$. Consider the region $W=\Omega-\cup_{1}^{\infty} \bar{Y}_{n}$ for a $\vartheta$-sequence $\left\{\bar{Y}_{n}\right\}$ and a density $P$ on $\Omega$ such that supp. $P \subset \cup_{1}^{\infty} \bar{Y}_{n}=\Omega-W$. We denote by $H_{u}^{W}$ for each $u$ in $P P(\Omega ; \partial \Omega)$ the least nonnegative harmonic function on $W$ with boundary values $u$ on $\partial W$ (cf. e.g. Constantinescu-Cornea [1]). It is the lower envelope of the family of superharmonic functions $s$ on $W$ with the lower limit boundary values of $s$ on $\partial W$ being not less than $u \mid \partial W$. Then the function $T_{P} u=u-H_{u}^{W}$ belongs to the class $H P(W ; \partial W)$ for every $u$ in $P P(\Omega ; \partial \Omega)$, and $u \mapsto T_{P} u$ defines a mapping $T_{P}: P P(\Omega ; \partial \Omega) \rightarrow H P(W ; \partial W)$. It is easy to see that the mapping $T_{P}$ is order preserving (i.e. $u_{1} \leqq u_{2}$ implies $T_{P} u_{1} \leqq T_{P} u_{2}$ ), positively homogeneous (i.e. $T_{P}(\lambda u)=\lambda T_{P}(u)$ for nonnegative real numbers $\lambda$ ), and additive (i.e. $\left.T_{P}\left(u_{1}+u_{2}\right)=T_{P} u_{1}+T_{P} u_{2}\right)$. In general $T_{P}$ may or may not be injective and similarly surjective. If the mapping $T_{P}$ happens to be bijective, then the density $P$ is said to be canonically associated with the $y$-sequence $\left\{\bar{Y}_{n}\right\}$. If a density $P$ on $\Omega$ is canonically associated with a $y$-sequence $\left\{\bar{Y}_{n}\right\}$, then we have

$$
\operatorname{dim} P=\operatorname{dim}\left\{\bar{Y}_{n}\right\} .
$$

To prove this we denote by $l(u)$ the left hand side of $(2)$. Then it is easy to see that $u \mapsto\left(2 \pi / l\left(T_{P} u\right)\right) T_{P} u$ is a bijective mapping of $P P_{1}(\Omega ; \hat{o} \Omega)$ onto $H P_{1}(W ; \partial W)$ along with $T_{P}$. We now prove the following

THEOREM. There always exists a density $P$ on $\Omega$ canonically associated with an arbitrarily given y-sequence $\left\{\bar{Y}_{n}\right\}$ in $\Omega$.

The proof of this assertion will be given in nos. 4-6 after establishing an auxiliary result in no. 3 .

3. We denote by $P_{f}^{U}$ the solution of (1) on the unit disk $U:|z|<1$ with boundary values $f$ on $\partial U$, where $P$ is a density on $\bar{U}$ and $f$ is in $C(\partial U)$. Give any Jordan region $V$ with $\bar{V} \subset U$ and any positive number $\varepsilon$. We then have the following simple but very useful fact (cf. [2]) :

Proposition. There exists a density $P=P_{V, s}$ on $\bar{U}$ with supp. $P \subset V$ and satisfying the inequality

$$
\sup _{V}\left|P_{f}^{U}\right| \leqq \frac{\varepsilon}{2 \pi} \int_{0}^{2 \pi}\left|f\left(e^{i \theta}\right)\right| d \theta
$$

for any $f$ in $C(\partial U)$.

For a proof of this we fix a disk $X:|z|<r(0<r<1)$ with $\bar{V} \subset X$. Since the Poisson kernel $P\left(e^{i \theta}, z\right)$ on $\partial U \times U$ is continuous on $\partial U \times \bar{X}$, there exists the maximum $c$ of $P\left(e^{i \theta}, z\right)$ on $\partial U \times \bar{X}$ :

$$
c=\max _{\partial U \times \bar{X}} P\left(e^{\imath \theta}, z\right) .
$$


Let $Y$ be an analytic Jordan region with $\bar{Y} \subset V$ and $\omega$ be the harmonic function on $X-\bar{Y}$ with boundary values $c$ on $\partial X, \varepsilon / 2$ on $\partial Y$. Taking $Y$ enough close to $V$ we can assume $\omega<\varepsilon$ on $\partial V$. Fix an analytic Jordan region $Z$ and a conformal mapping $\zeta=\phi(z): \bar{Z} \rightarrow \phi(\bar{Z})=\{|\zeta| \leqq 1\}$ such that $\bar{Y} \subset Z, \bar{Z} \subset V$, and $\phi(Y)$ is a disk with center at $\zeta=0$. Consider the density $\phi_{m}$ and the function $v_{m}$ on $\phi(\bar{Z})$ defined by

$$
\phi_{m}(\zeta)=\frac{4 m^{2}|\zeta|^{2 m-2}}{|\zeta|^{2 m}+(2 m)^{-1}}, \quad v_{m}(\zeta)=\frac{|\zeta|^{2 m}+(2 m)^{-1}}{1+(2 m)^{-1}} \quad(m=1,2, \cdots)
$$

and observe that

$$
4 \frac{\partial^{2}}{\partial \zeta \partial \bar{\zeta}} v_{m}(\zeta)=\phi_{m}(\zeta) v_{m}(\zeta) \quad(|\zeta|<1)
$$

If we set

$$
Q_{m}(z)=\phi_{m}(\phi(z))\left|\phi^{\prime}(z)\right|^{2}, \quad w_{m}(z)=v_{m}(\phi(z))
$$

the function $w_{m}$ on $Z$ has boundary values 1 on $\partial Z$ and satisfies

$$
4 \frac{\partial^{2}}{\partial z \partial \bar{z}} u_{m}(z)=Q_{m}(z) w_{m}(z)
$$

on $Z$. We take a density $P_{m}$ on $\bar{U}$ with supp. $P_{m} \subset V, P_{m} \geqq Q_{m}$ on $\bar{Z}$ and $P_{m} \leqq P_{m+1}$ on $\bar{U}(m=1,2, \cdots)$. Since $P_{m} \geqq Q_{m}$ on $\bar{Z}$, the solution $u_{m}$ of

$$
4-\frac{\partial^{2}}{\partial z \hat{\partial} \bar{z}} u(z)=P_{m}(z) u(z)
$$

on $X$ with boundary values $c$ on $\partial X$ satisfies $u_{m} \leqq c w_{m}$ on $\bar{Z}$. Observe that $\left\{w_{m}\right\}$ converges to 0 uniformly on $\bar{Y}$ as $m \rightarrow \infty$. Then for some positive integer $m_{\varepsilon}$ we have $u_{m_{\varepsilon}}<\varepsilon / 2$ on $\partial Y$ so that $u_{m_{\varepsilon}} \leqq \omega$ on $X-\bar{Y}$. Therefore $u_{m_{\varepsilon}}<\varepsilon$ on $\partial V$, and hence on $\bar{V}$. Now we set

$$
P=P_{V, s}=P_{m_{\varepsilon}}, \quad v=u_{m_{\varepsilon}} .
$$

We denote by $g(\zeta, z), G(\zeta, z)$ the harmonic Green's function, $P$-Green's function on $U$, respectively. We remark that $G(\zeta, z) \leqq g(\zeta, z)$ and

$$
P\left(e^{i \theta}, z\right)=\left[-\frac{\partial}{\partial|\zeta|} g(\zeta, z)\right]_{\zeta=e^{2 \theta}}
$$

for $e^{2 \theta}$ in $\partial U$ and $z$ in $U$. If we set

$$
K\left(e^{i \theta}, z\right)=\left[-\frac{\partial}{\partial|\zeta|} G(\zeta, z)\right]_{\zeta=e^{\imath \theta}},
$$

then we have $0<K\left(e^{\imath \theta}, z\right) \leqq P\left(e^{i \theta}, z\right)$. Since $P\left(e^{i \theta}, z\right) \leqq c$ on $\partial U \times \bar{X} v(z)=c$ on $\partial X$ we have $K\left(e^{i \theta}, z\right) \leqq \nu(z)$ on $X$ so that $K\left(e^{i \theta}, z\right)<\varepsilon$ on $\partial U \times \bar{V}$. Thus we have for $f$ in $C(\partial U)$ and $z$ in $\bar{Y}$ 


$$
\begin{aligned}
\left|P_{f}^{U}(z)\right| & =\left|\frac{1}{2 \pi} \int_{0}^{2 \pi} f\left(e^{i \theta}\right) K\left(e^{i \theta}, z\right) d \theta\right| \\
& \leqq \frac{1}{2 \pi} \int_{0}^{2 \pi}\left|f\left(e^{i \theta}\right)\right| K\left(e^{i \theta}, z\right) d \theta \\
& \leqq \frac{\varepsilon}{2 \pi} \int_{0}^{2 \pi}\left|f\left(e^{i \theta}\right)\right| d \theta .
\end{aligned}
$$

4. We proceed to the proof of Theorem in no. 2, i.e. the existence of canonically associated densities. Let $\left\{\bar{Y}_{n}\right\}$ be any $q$-sequence on $\Omega$ and $U_{n}$ be a slightly larger analytic Jordan region in $\Omega$ than $Y_{n}$ containing $\bar{Y}_{n}$. We may assume $\bar{U}_{n} \cap \bar{U}_{m}=\varnothing$ for $n \neq m$. We fix a point $z_{1}$ in $\Omega-\cup_{1}^{\infty} \bar{U}_{n}$ and denote by $F$ the set of nonnegative harmonic functions $u$ on $W=\Omega-\bigcup_{1}^{\infty} \bar{Y}_{n}$ with $u\left(z_{1}\right)=1$. Then the Harnack principle yields for every nonnegative integer $n$

$$
b_{n} \equiv \sup _{F} \max _{\partial U_{n}} h<+\infty .
$$

Using a conformal mapping $\phi_{n}$ of $\bar{U}_{n}$ to $\phi_{n}\left(\bar{U}_{n}\right)=\{|z| \leqq 1\}$ we define a density $P=P_{W}$ on $\Omega$ by

$$
P(\zeta)= \begin{cases}P_{n}\left(\dot{\phi}_{n}(\zeta)\right) & \left(\zeta \in \bar{U}_{n} ; n=1,2, \cdots\right) \\ 0 & \left(\zeta \in \Omega-\cup_{1}^{\infty} \bar{U}_{n}\right)\end{cases}
$$

where $P_{n}$ is a density on $\bar{U}=\{|z|<1\}$ which satisfies (7) for $V=\phi_{n}\left(Y_{n}\right)$, $\varepsilon=$ $1 / n\left(b_{n}+1\right)$. We will show that $P$ is a canonically associated density with $\left\{\bar{Y}_{n}\right\}$.

5. First we prove that the mapping $T_{P}$ is injective. Let $u_{1}, u_{2}$ be functions in $P P(\Omega ; \partial \Omega)$ with $T_{P} u_{1}=T_{P} u_{2}$. Then we have $u_{1}-u_{2}=H_{u_{1}-u_{2}}^{W}$ on $W$. Assume that $u_{1} \neq \equiv u_{2}$. Then we may assume $\sup _{W}\left(u_{1}-u_{2}\right)>0$ if necessary by exchanging indices of $u_{1}, u_{2}$. Since $H_{u_{1}-u_{2}}^{W}$ is quasibounded we have $\sup _{W} H_{u_{1}-u_{2}}^{W}=\sup _{\partial W} H_{u_{1}-u_{2}}^{W}$. We set $v_{k}=u_{k} /\left(u_{k}\left(z_{1}\right)+1\right)(k=1,2)$. Then $v_{k}$ satisfies $v_{k} \leqq b_{n}$ on $\partial U_{n}$ so that we have for $z$ in $\bar{Y}_{n}$

$$
v_{k}(z) \leqq \frac{1}{2 \pi n\left(b_{n}+1\right)} \int_{0}^{2 \pi} v_{k}\left(\phi_{n}^{-1}\left(e^{i \theta}\right)\right) d \theta \leqq \frac{1}{n} .
$$

Then we have $H_{u_{1}-u_{2}}^{W}=u_{1}-u_{2} \leqq\left(u_{1}\left(z_{1}\right)+u_{2}\left(z_{1}\right)+2\right) / n$ on $\partial Y_{n}$, and hence the harmonic function $H_{u_{1}-u_{2}}^{W}$ on $W$ attains its supremum at a point in some $\partial Y_{n}$. Therefore the function $u_{1}-u_{2}$ in $P P(\Omega ; \partial \Omega)$ attains its maximum at the same point as that of $H_{u_{1}-u_{2}}^{W}$ so that $u_{1}-u_{2}$ is identically a positive constant. This contradicts the fact that $u_{1}-u_{2} \leqq\left(u_{1}\left(z_{1}\right)+u_{2}\left(z_{1}\right)+2\right) / n$ on $\bar{Y}_{n}$ for every positive integer $n$. Thus $u_{1} \equiv u_{2}$.

6. Next we prove that $T_{P}$ is surjective. Let $h$ be any function in $H P(W ; \partial W)$. Since $T_{P}$ is positively homogeneous we may assume that $h$ is in $F$. We denote by $w$ the harmonic measure of $\partial W-\partial \Omega$ considered on $W$ and we set $h_{1}=h+w$ 
on $W$. Observe that for $z$ in $\partial Y_{n}$

$$
\begin{aligned}
P_{h_{1}}^{U n}(z) & \leqq \frac{1}{2 \pi n\left(b_{n}+1\right)} \int_{0}^{2 \pi} h_{1}\left(\phi_{n}^{-1}\left(e^{i \theta}\right)\right) d \theta \\
& \leqq \frac{b_{n}+1}{n\left(b_{n}+1\right)} \leqq 1=h_{1}(z) .
\end{aligned}
$$

Then we have $P_{h_{1}}^{C_{n}} \leqq h_{1}$ on $U_{n}-\bar{Y}_{n}$ by the maximum principle. Therefore the function $s$ on $\Omega$ defined by $s=h_{1}$ on $\Omega-\bigcup_{1}^{\infty} U_{n}, s=P_{h_{1}^{n}}^{U_{n}}$ on $U_{n}(n=1,2, \cdots)$ is a supersolution of (1) (i.e. superharmonic with respect to (1)) on $\Omega$ with $h \leqq s \leqq h_{1}$ on $W$. On the other hand $h$ is a subsolution of (1) (i.e. subharmonic with respect to (1)) on $\Omega$ if we define $h=0$ on $\cup_{1}^{\infty} Y_{n}$. Now the lower envelope $u$ of the family of supersolutions of (1) on $\Omega$ which dominate $h$ on $\Omega$ is a solution of (1) with $h \leqq u$ on $\Omega, u \leqq h_{1}$ on $W$ so that $u$ is in $P P(\Omega ; \partial \Omega)$. If we set $v=-h+u-H_{u}^{W}$ on $W, v$ satisfies $v \leqq-h+u \leqq-h+h_{1}=w \leqq 1, v \geqq-H_{u}^{W} \geqq-H_{h_{1}}^{W}=-w \geqq-1$. On the other hand $v$ vanishes on $\partial W$ and therefore $v \equiv 0$, i.e. $T_{P} u=h$. This completes the proof of Theorem in no. 2 .

\section{$\S 2$. Relative harmonic dimension.}

7. In view of (6) and the theorem in no. 2, the proof of the main theorem is reduced to showing that $\operatorname{dim} y(\Omega)$ contains $\Xi$. In passing we remark that $y(\Omega) \subset \mathcal{K}(\Omega)$ implies that $\operatorname{dim} \mathcal{K}(\Omega)$ contains $\Xi$ along with $\operatorname{dim} y(\Omega)$. Thus the proof of the main theorem will be complete if we show the following fact which may have an independent interest in its own right:

THEOREM. There exists a as-sequence $\left\{\bar{Y}_{n}\right\}$ in $\Omega$ for any cardinal number $m$ in $\Xi$ such that $\operatorname{dim}\left\{\bar{Y}_{n}\right\}=\mathfrak{m}$.

Therefore we have $\Xi \subset \operatorname{dim} y(\Omega) \subset \operatorname{dim} \mathcal{K}(\Omega) \subset[1, \mathrm{c}]$ so that $\operatorname{dim} y(\Omega)=$ $\operatorname{dim} \mathcal{K}(\Omega)=\Xi$ if we assume the continuum hypothesis $\Xi=[1, \mathrm{c}]$. The proof will be given in nos. 8-14 by exhibiting three examples in nos. 9, 12, and 14 .

8. Before proceeding to our three examples we remark the following simple fact (cf. e.g. Constantinescu-Cornea [1]) which plays an important role in verifying that the examples in nos. 9,12 , and 14 are required ones. Let $\left\{U_{n}\right\}_{1}^{\infty}$ be a sequence of relatively compact subregion $U_{n}$ in $\Omega$ such that $\bar{U}_{n} \cap \bar{U}_{m}=\varnothing(n \neq m)$ and $\left\{\bar{U}_{n}\right\}$ converges to $\delta \Omega: z=0$. Consider a region $W=\Omega-\cup_{1}^{\infty} K_{n}$ for a $\mathcal{K}$-sequence $\left\{K_{n}\right\}$ in $\Omega$. Set $V_{n}=W \cap U_{n}$ and $V=\cup_{1}^{\infty} V_{n}$. Let $\delta W, \delta_{1} W$ be the Martin boundary of $W$ over $\delta \Omega: z=0$, the set of minimal points in $\delta W$, respectively and $(W-V)^{a}$ the closure of $W-V$ considered in the Martin compactification of $W$. Then

$$
\delta W-(W-V)^{a} \subset \delta W-\delta_{1} W
$$


9. ExAmple 1. First we exhibit an example of a $y$-sequence $\left\{Y_{n}\right\}_{1}^{\infty}$ with $\operatorname{dim}\left\{\bar{Y}_{n}\right\}=m$ for any given positive integer $m$. Fix a sequence $\left\{a_{n}\right\}_{1}^{\infty}$ in $(0,1)$ with $a_{n+1}<a_{n}(n=1,2, \cdots)$ and $\lim a_{n}=0$. Fix a positive numbers $\theta_{1}, \cdots, \theta_{m}$; $\eta_{1}, \cdots, \eta_{m}$ with $\theta_{\mu}+2 \eta_{\mu}<-2 \eta_{\mu+1}+\theta_{\mu+1}(\mu=0,1, \cdots, m)$, where $\theta_{0}+2 \eta_{0}=0$, $-2 \eta_{m+1}+\theta_{m+1}=2 \pi$. We choose a sequence $\left\{b_{n}\right\}_{1}^{\infty}$ in $(0,1)$ with $a_{n+1}<b_{n}<a_{n}$. Let (see Fig. 1)

$$
S_{n \mu}=\left\{b_{n}<|z|<a_{n},\left|\arg z-\theta_{\mu}\right|<\eta_{\mu}\right\} \quad(\mu=1, \cdots, m ; n=1,2, \cdots) .
$$

Observe that any positive integer $k$ has a unique expression $k=(n-1) m+\mu$ with positive integers $n$ and $\mu$ with $1 \leqq \mu \leqq m$. We set

$$
Y_{k}=S_{n \mu} \quad(k=(n-1) m+\mu) .
$$

Then the sequence $\left\{\bar{Y}_{k}\right\}_{1}^{\infty}=\left\{\bar{S}_{n \mu}\right\}$ is clearly a $y$-sequence. If we choose the sequence $\left\{b_{n}\right\}$ so as to make the sequence $\left\{b_{n}-a_{n+1}\right\}_{1}^{\infty}$ converges to zero enough rapidly, i.e. satisfying (9), (11), and (14) below then we can show that $\operatorname{dim}\left\{\bar{S}_{n \mu}\right\}$ $=m$ in the following way.

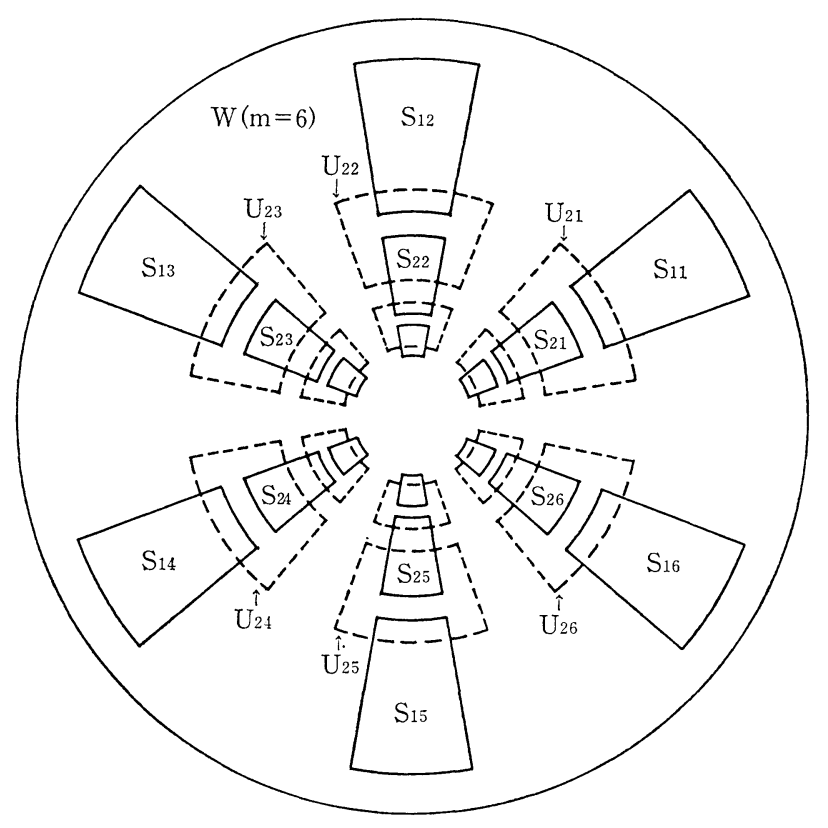

Fig. 1.

10. Fix a sequence $\left\{\delta_{n}\right\}_{2}^{\infty}$ in $(0,1)$ with $a_{n+1}+\delta_{n+1}<a_{n}-\delta_{n} \quad(n=1,2, \cdots)$, where $\delta_{1}=0$. We set (see Fig. 1)

$$
U_{n \mu}=\left\{|| z\left|-a_{n}\right|<\delta_{n},\left|\arg z-\theta_{\mu}\right|<2 \eta_{\mu}\right\} \quad(\mu=1, \cdots, m ; n=2,3, \cdots)
$$


and $U=\bigcup_{n=2}^{\infty} \bigcup_{\mu=1}^{m} U_{n \mu}$. Then we have $\bar{U}_{n \mu} \cap \bar{U}_{k \nu}=\varnothing((n, \mu) \neq(k, \nu))$. The first property which $\left\{b_{n}\right\}$ has to satisfy is

$$
b_{n}<a_{n+1}+\delta_{n+1} \quad(n=1,2, \cdots) .
$$

We consider subregions $W$ and $W_{n}(n=1,2, \cdots)$ of $\Omega$ given by

$$
\begin{gathered}
W=\Omega-\bigcup_{n=1}^{\infty} \bigcup_{\mu=1}^{m} \bar{S}_{n \mu}, \\
W_{n}=W-\bigcup_{\mu=1}^{m}\left\{0<|z| \leqq a_{n},\left|\arg z-\theta_{\mu}\right| \leqq \eta_{\mu}\right\}
\end{gathered}
$$

and denote by $g(\zeta, z), g_{n}(\zeta, z)$ the Green's functions on $W, W_{n}$, respectively. Fix a reference point $a$ of the Martin kernels $k(\zeta, z)=g(\zeta, z) / g(\zeta, a), k_{n}(\zeta, z)=$ $g_{n}(\zeta, z) / g_{n}(\zeta, a)$ on $W, W_{n}$, respectively and a neighbourhood $D$ of $a$ with $\bar{D} \subset W-\bar{U}$. Finally we fix a sequence $\left\{\varepsilon_{n}\right\}_{1}^{\infty}$ in $(0,1)$ with

$$
\sum_{n=1}^{\infty} \varepsilon_{n}<1, \quad \prod_{n=1}^{\infty}\left(1-\varepsilon_{n}\right) \geqq \frac{1}{2} .
$$

Then the second property which $\left\{b_{n}\right\}$ has to satisfy is

$$
g_{n+1}(\zeta, z)-g_{n}(\zeta, z) \leqq \varepsilon_{n} g_{n}(\zeta, z)
$$

for any $z$ in $D$ and $\zeta$ in $W-U$. Assume (11) is valid for every positive integer $n$. Then we have

so that

$$
g_{n+j}(\zeta, z)-g_{n}(\zeta, z) \leqq \alpha_{n} g_{n+j}(\zeta, z)
$$

$$
\left\{\begin{array}{l}
g_{n}(\zeta, z) \leqq g_{n+j}(\zeta, z) \leqq\left(1-\alpha_{n}\right)^{-1} g_{n}(\zeta, z) \\
g_{n}(\zeta, z) \leqq g(\zeta, z) \leqq\left(1-\alpha_{n}\right)^{-1} g_{n}(\zeta, z)
\end{array}\right.
$$

for every positive integer $j$, where $\alpha_{n}=\sum_{k=n}^{\infty} \varepsilon_{k}$. Therefore Martin kernels $k$, $k_{n}$ satisfy on $(W-U) \times D$

$$
\begin{aligned}
& \left(1-\alpha_{n}\right) k_{n}(\zeta, z) \leqq k_{n+j}(\zeta, z) \leqq\left(1-\alpha_{n}\right)^{-1} k_{n}(\zeta, z), \\
& \left(1-\alpha_{n}\right) k_{n}(\zeta, z) \leqq k(\zeta, z) \leqq\left(1-\alpha_{n}\right)^{-1} k_{n}(\zeta, z) .
\end{aligned}
$$

Let $\delta W_{n}$ and $\delta_{1} W_{n}$ be the Martin boundary of $W_{n}$ over $\delta \Omega: z=0$ and the set of minimal points in $\delta W_{n}$, respectively. Then we have $\delta W_{n}=\delta_{1} W_{n}=\delta W_{1}=\delta_{1} W_{1}=$ $\left\{p_{1}, \cdots, p_{m}\right\}$ and we may assume for every positive integer $n, \mu, \nu$ with $1 \leqq \mu, \nu \leqq m$

$$
\lim _{\Gamma_{\mu \ni z \rightarrow 0}} k_{n}\left(p_{\nu}, z\right)=\left\{\begin{array}{ll}
+\infty & (\mu=\nu) \\
0 & (\mu \neq \nu)
\end{array},\right.
$$

where $\Gamma_{\mu}=\left\{0<|z|<1, \quad\right.$ arg $\left.z=\left(\theta_{\mu}+2 \eta_{\mu}+\theta_{\mu+1}-2 \eta_{\mu+1}\right) / 2\right\}$ and $\theta_{m+1}-2 \eta_{m+1}=$ $\theta_{1}-2 \eta_{1}$. Making $\zeta \rightarrow p_{\mu}$ in (12) we have 


$$
\left(1-\alpha_{n}\right) k_{n}\left(p_{\mu}, z\right) \leqq k_{n+j}\left(p_{\mu}, z\right) \leqq\left(1-\alpha_{n}\right)^{-1} k_{n}\left(p_{\mu}, z\right)
$$

for every positive integer $\mu$ with $1 \leqq \mu \leqq m$. Then the following limit exists for any $z$ in $D: A_{\mu}(z)=\lim _{n \rightarrow \infty} k_{n}\left(p_{\mu}, z\right)$, and hence $\left\{k_{n}\left(p_{\mu}, z\right)\right\}_{n=1}^{\infty}$ converges to a nonnegative harmonic function $A_{\mu}$ on $W$ uniformly on every compact subset of $W$. On the other hand from (13) it follows that harmonic functions

on $W$ satisfy

$$
\bar{A}_{\mu}(z)=\varlimsup_{\zeta \rightarrow p_{\mu}} k(\zeta, z), \quad \underline{A}_{\mu}(z)=\lim _{\zeta \rightarrow p_{\mu}} k(\zeta, z)
$$

$$
\left(1-\alpha_{n}\right) k_{n}\left(p_{\mu}, z\right) \leqq \underline{A}_{\mu}(z) \leqq \bar{A}_{\mu}(z) \leqq\left(1-\alpha_{n}\right)^{-1} k_{n}\left(p_{\mu}, z\right)
$$

on $D$. By making $n \rightarrow \infty$ in the above inequalities we have $A_{\mu}(z)=\underline{A}_{\mu}(z)=\bar{A}_{\mu}(z)$ on $D$, and hence on $W$. Then every $p_{\mu}$ defines unique Martin boundary point $q_{\mu}$ of $W$ over $\delta \Omega: z=0$ such that

$$
\lim _{n \rightarrow \infty} k_{n}\left(p_{\mu}, z\right)=\lim _{\zeta \rightarrow p_{\mu}} k(\zeta, z)=k\left(q_{\mu}, z\right)
$$

on $W$. We remark that it may happen $q_{\mu}=q_{\nu}$ for some $\mu$, $\nu$ with $\mu \neq \nu$. Let $q$ be any point in $(W-U)^{a} \cap \delta W$. Then there exists a sequence $\left\{\zeta_{n}\right\}_{1}^{\infty}$ in $W-U$ with $\zeta_{n} \rightarrow q$. Since a subsequence $\left\{\zeta_{n}^{\prime}\right\}_{1}^{\infty}$ of $\left\{\zeta_{n}\right\}$ converges to a point $p_{\mu}$ in $\delta \Omega_{1}$ we have $q=q_{\mu}$ so that $\delta W-(W-U)^{a}$ contains $\delta W-\left\{q_{\mu}\right\}_{1}^{m}$. Therefore by (8) $\delta W-\delta_{1} W$ contains $\delta W-\left\{q_{\mu}\right\}_{1}^{m}$, and hence we have $\delta_{1} W \subset\left\{q_{\mu}\right\}_{1}^{m}$. Thus we conclude that $\operatorname{dim}\left\{\bar{S}_{n \mu}\right\} \leqq m$.

11. Now we give the last property which $\left\{b_{n}\right\}$ has to satisfy. Consider the harmonic function $u_{n \mu}$ on $W_{n-1}$ with boundary values $k_{n}\left(p_{\mu}, z\right)$ on $\partial W_{n-1}$ $(\mu=1, \cdots, m ; n=2,3, \cdots)$. We require $\left\{b_{n}\right\}$ to satisfy

$$
u_{n+1, \mu}(z) \leqq \varepsilon_{n}
$$

on $W-U$ for every $\mu$. Since the nonnegative harmonic function $k_{n+1}\left(p_{\mu}, z\right)-$ $u_{n+1, \mu}(z)$ vanishes on $\partial W_{n}$ it is represented by $k_{n}\left(p_{\mu}, z\right)$ :

$$
k_{n+1}\left(p_{\mu}, z\right)-u_{n+1, \mu}(z)=\left(1-u_{n+1, \mu}(a)\right) k_{n}\left(p_{\mu}, z\right) .
$$

Assume (14) is valid for every positive integer $n$. Then if we set $v_{1 \mu}(z)=k_{1}\left(p_{\mu}, z\right.$ and

$$
v_{n \mu}(z)=\left(\prod_{k=2}^{n}\left(1-u_{k \mu}(a)\right)\right)^{-1} k_{n}\left(p_{\mu}, z\right) \quad(\mu=1, \cdots, m ; n=2,3, \cdots),
$$

by (10) and (14) we have

$$
\begin{aligned}
\left|v_{n+1, \mu}(z)-v_{n \mu}(z)\right| & =\left(\prod_{k=2}^{n+1}\left(1-u_{k \mu}(a)\right)\right)^{-1} \mid k_{n+1}\left(p_{\mu}, z\right)-\left(1-u_{n+1, \mu}(a)\right) k_{n}\left(p_{\mu}, z\right) \\
& \leqq\left(\prod_{k=1}^{\infty}\left(1-\varepsilon_{k}\right)\right)^{-1} \varepsilon_{n} \leqq 2 \varepsilon_{n}
\end{aligned}
$$


on $W-U$. Therefore $\left\{v_{n \mu}\right\}_{n=1}^{\infty}$ converges to a nonnegative harmonic function $v_{\mu}$ in $H P(W ; \partial W)$ uniformly on every compact subset of $W$. Since we have

$$
\left|v_{n+j, \mu}-v_{n \mu}\right| \leqq 2\left(\varepsilon_{n}+\cdots+\varepsilon_{n+j-1}\right)
$$

for every positive integer $j$, every function $v_{\mu}$ satisfies $v_{n \mu}-2 \alpha_{n} \leqq v_{\mu} \leqq v_{n \mu}+2 \alpha_{n}$ on $W-U$. Then $v_{\mu}$ has the same limit as that of $v_{n \mu}$ at $\delta \Omega: z=0$ along $\Gamma_{\nu}$ :

$$
\lim _{\Gamma_{\nu} \ni z \rightarrow 0} v_{\mu}(z)=\left\{\begin{array}{ll}
+\infty & (\mu=\nu) \\
0 & (\mu \neq \nu)
\end{array} .\right.
$$

Thus we have $\operatorname{dim}\left\{\bar{S}_{n \mu}\right\} \geqq m$.

12. EXAMPLE 2. Next we exhibit an example of a $y$-sequence $\left\{\bar{Y}_{n}\right\}_{1}^{\infty}$ with $\operatorname{dim}\left\{\bar{Y}_{n}\right\}=a$. Fix a sequence $\left\{a_{n}\right\}_{1}^{\infty}$ in $(0,1)$ with $a_{n+1}<a_{n}(n=1,2, \cdots)$ and $\lim a_{n}=0$. Fix sequences $\left\{\theta_{\mu}\right\}_{1}^{\infty},\left\{\eta_{\mu}\right\}_{1}^{\infty}$ of positive numbers $\theta_{\mu}, \eta_{\mu}$ with $0<\theta_{1}-2 \eta_{1}$, $\theta_{\mu}+2 \eta_{\mu}<-2 \eta_{\mu+1}+\theta_{\mu+1}<2 \pi$ for every positive integer $\mu$. We choose a sequence $\left\{b_{n}\right\}_{1}^{\infty}$ in $(0,1)$ with $a_{n+1}<b_{n}<a_{n}(n=1,2, \cdots)$. Let

$$
S_{n \mu}=\left\{b_{n}<|z|<a_{n},\left|\arg z-\theta_{\mu}\right|<\eta_{\mu}\right\} \quad(\mu=1, \cdots, n ; n=1,2, \cdots) .
$$

Observe that any positive integer $k$ has a unique expression $k=n(n-1) / 2+\mu$ with positive integers $n$ and $\mu$ satisfying $n \geqq \mu$. We set (see Fig. 2)

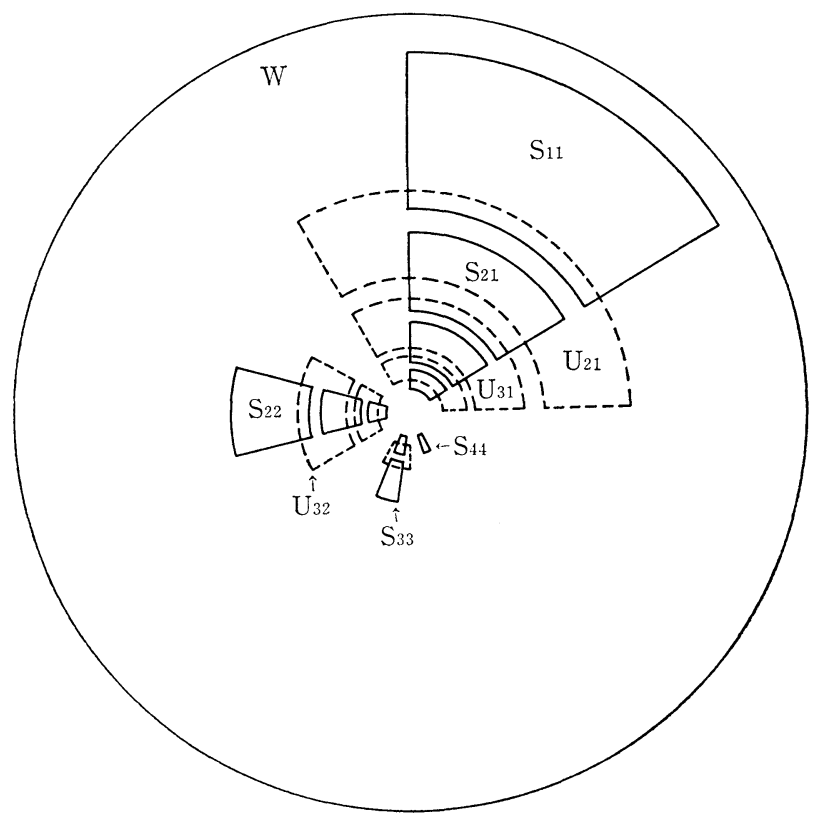

Fig. 2. 


$$
Y_{k}=S_{n \ell} \quad(k=n(n-1) / 2+\mu) .
$$

Then the sequence $\left\{\bar{Y}_{k}\right\}_{1}^{\infty}=\left\{\bar{S}_{n \mu}\right\} \quad(\mu=1, \cdots, n ; n=1,2, \cdots)$ is a $y$-sequence. If we choose the sequence $\left\{b_{n}\right\}$ so as to make the sequence $\left\{b_{n}-a_{n+1}\right\}_{1}^{\infty}$ converges to zero enough rapidly, i.e. satisfying (15), (16), and (17) below then we can show that $\operatorname{dim}\left\{\bar{S}_{n \mu}\right\}=\mathfrak{a}$ in the similar way as in nos. 10 and 11 .

13. Fix a sequence $\left\{\delta_{n}\right\}_{2}^{\infty}$ in $(0,1)$ with $a_{n+1}+\delta_{n+1}<a_{n}-\delta_{n}(n=1,2, \cdots)$, where $\delta_{1}=0$. We set (see Fig. 2)

$$
U_{n \mu}=\left\{|| z\left|-a_{n}\right|<\delta_{n},\left|\arg z-\theta_{\mu}\right|<2 \eta_{\mu}\right\} \quad(\mu=1, \cdots, n-1 ; n=2,3, \cdots)
$$

and $U=\bigcup_{n=2}^{\infty} \bigcup_{\mu=1}^{n-1} U_{n \mu}$. Then we have $\bar{U}_{n \mu} \cap \bar{U}_{k \nu}=\varnothing((n, \mu) \neq(k, \nu))$. The first property which $\left\{b_{n}\right\}$ has to satisfy is

$$
b_{n}<a_{n+1}+\delta_{n+1} \quad(n=1,2, \cdots) .
$$

We consider subregions $W$ and $W_{n}(n=1,2, \cdots)$ of $\Omega$ given by

$$
\begin{gathered}
W=\Omega-\bigcup_{n=1}^{\infty} \bigcup_{\mu=1}^{n} \bar{S}_{n \mu}, \\
W_{n}=W-\bigcup_{\mu=1}^{n}\left\{0<|z| \leqq a_{n},\left|\arg z-\theta_{\mu}\right| \leqq \eta_{\mu}\right\} \\
-\bigcup_{\mu=n+1}^{\infty}\left\{0<|z| \leqq a_{\mu},\left|\arg z-\theta_{\mu}\right| \leqq \eta_{\mu}\right\}
\end{gathered}
$$

and denote by $g(\zeta, z), g_{n}(\zeta, z)$ the Green's functions on $W, W_{n}$, respectively. Fix a reference point $a$ of the Martin kernels $k(\zeta, z)=g(\zeta, z) / g(\zeta, a), k_{n}(\zeta, z)=$ $g_{n}(\zeta, z) / g_{n}(\zeta, a)$ on $W, W_{n}$, respectively and a neighbourhood $D$ of $a$ with $\bar{D} \subset W-\bar{U}$. Finally we fix a sequence $\left\{\varepsilon_{n}\right\}_{1}^{\infty}$ in $(0,1)$ with $\sum_{1}^{\infty} \varepsilon_{n}<1, \Pi_{1}^{\infty}\left(1-\varepsilon_{n}\right)$ $\geqq 1 / 2$. Then the second property which $\left\{b_{n}\right\}$ has to satisfy is

$$
g_{n+1}(\zeta, z)-g_{n}(\zeta, z) \leqq \varepsilon_{n} g_{n}(\zeta, z)
$$

for any $z$ in $D$ and $\zeta$ in $W-U$. Let $\delta W_{n}$ and $\delta_{1} W_{n}$ be the Martin boundary over $\delta \Omega: z=0$ and the set of minimal points in $\delta W_{n}$, respectively. Then we have $\delta W_{n}=\delta_{1} W_{n}=\delta W_{1}=\delta_{1} W_{1}=\left\{p_{1}, p_{2}, \cdots\right.$; and $\left.p_{\infty}\right\}$ and we may choose a family $\left\{\Gamma_{\mu}: \mu=1,2, \cdots\right.$; and $\left.\infty\right\}$ of pairwize disjoint curves $\Gamma_{\mu}$ in $W-\bar{U}$ converging to $\delta \Omega: z=0$ such that

$$
\lim _{\Gamma_{\mu} \ni z \rightarrow 0} k_{n}\left(p_{2}, z\right)= \begin{cases}+\infty & (\mu=\nu) \\ 0 & (\mu \neq \nu)\end{cases}
$$

for every positive integer $n$. Assume (16) is valid for every $n$. Then $k_{n}\left(p_{\mu}, z\right)$ and $k(\zeta, z)$ converge to a same function in $H P(W ; \partial W)$ uniformly on every compact subset of $W$ as $n \rightarrow \infty$ and $\zeta \rightarrow p_{\mu}$, respectively for every $\mu=1,2, \cdots$; and $\infty$. Therefore every $p_{\mu}$ defines unique Martin boundary point $q_{\mu}$ of $W$ over $\delta \Omega$ : 
$z=0$ such that

$$
\lim _{n \rightarrow \infty} k_{n}\left(p_{\mu}, z\right)=\lim _{\zeta \rightarrow p_{\mu}} k(\zeta, z)=k\left(q_{\mu}, z\right)
$$

on $W$. We remark that it may happen $q_{\mu}=q_{\nu}$ for some $\mu, \nu$ with $\mu \neq \nu$. We denote by $\delta W$ and $\delta_{1} W$ the Martin boundary of $W$ over $\delta \Omega: z=0$ and the set of minimal points in $\delta W$, respectively. Observe that $\delta W-(W-U)^{a}$ contains $\delta W-\left\{q_{1}, q_{2}, \cdots\right.$; and $\left.q_{\infty}\right\}$. Then by (8) $\delta W-\delta_{1} W$ contains $\delta W-\left\{q_{1}, q_{2}, \cdots\right.$; and $\left.q_{\infty}\right\}$ and hence $\delta_{1} W \subset\left\{q_{1}, q_{2}, \cdots\right.$; and $\left.q_{\infty}\right\}$. Thus we conclude that $\operatorname{dim}\left\{\bar{S}_{n \mu}\right\} \leqq \mathfrak{a}$.

We give the last property which $\left\{b_{n}\right\}$ has to satisfy. Consider the harmonic function $u_{n \mu}$ on $W_{n-1}$ with boundary values $k_{n}\left(p_{\mu}, z\right)$ on $\partial W_{n-1}$ for $n=2,3, \cdots$ and $\mu=1,2, \cdots$; and $\infty$. We requir $\left\{b_{n}\right\}$ to satisfy

$$
u_{n+1, \mu}(z) \leqq \varepsilon_{n}
$$

on $W-U$ for every $\mu$. We set $v_{1 \mu}(z)=k_{1}\left(p_{\mu}, z\right)$ and

$$
v_{n \mu}(z)=\left(\prod_{k=2}^{n}\left(1-u_{k \mu}(a)\right)\right)^{-1} k_{n}\left(p_{\mu}, z\right) \quad(n=2,3, \cdots ; \mu=1,2, \cdots ; \text { and } \infty) .
$$

Assume (17) is valid. Then $v_{n_{\mu}}$ converges to a function $v_{\mu}$ in $H P(W ; \partial W)$ uniformly on every compact subset of $W$ and $v_{\mu}$ has the same limit as that of $k_{n}\left(p_{\mu}, z\right)$ at $\delta \Omega: z=0$ along $\Gamma_{\nu}$ :

$$
\lim _{\Gamma_{\nu} \ni z \rightarrow 0} v_{\mu}(z)= \begin{cases}+\infty & (\mu=\nu) \\ 0 & (\mu \neq \nu)\end{cases}
$$

for $\mu, \nu=1,2, \cdots$; and $\infty$. Thus we have $\operatorname{dim}\left\{\bar{S}_{n \mu}\right\} \geqq \mathfrak{a}$.

14. EXAMPLE 3. Finally we exhibit an example of a $y$-sequence $\left\{\bar{Y}_{n}\right\}_{1}^{\infty}$ with $\operatorname{dim}\left\{\bar{Y}_{n}\right\}=$ c. Fix a sequence $\left\{a_{n}\right\}_{1}^{\infty}$ in $(0,1)$ with $a_{n+1}<a_{n}(n=1,2, \cdots)$ and $\lim a_{n}=0$. Fix a sequence $\left\{\eta_{\mu}\right\}_{0}^{\infty}$ of positive numbers $\eta_{\mu}$ with

$$
4\left(\eta_{0}+\sum_{\mu=1}^{\infty} 2^{\mu-1} \eta_{\mu}\right)<2 \pi \text {. }
$$

For every positive integer $n$ we set $\eta_{n 0}=\eta_{0}$ and for every positive integer $\mu$ with $\mu \leqq 2^{n}-1$ we set $\eta_{n \mu}=\eta_{n-j}$ if $\mu=k 2^{j}$ for positive odd integer $k$. Then we define a sequence $\left\{\theta_{n \mu}\right\}\left(0 \leqq \mu \leqq 2^{n}-1, n \geqq 1\right)$ in $[0,2 \pi)$ by induction : $\theta_{10}=0, \theta_{11}=\pi$; and in the case $n \geqq 2$ we set

$$
\theta_{n \mu}=\theta_{n-1, \mu / 2},
$$

if $\mu$ is a nonnegative even integer,

$$
\begin{aligned}
\theta_{n \ell}= & \frac{1}{2}\left(\theta_{n-1,(\mu 1) / 2}+2 \eta_{n 1,(\mu-1) / 2}\right. \\
& \left.-2 \eta_{n-1,(\mu+1) / 2}+\theta_{n-1,(\mu+1) / 2}\right),
\end{aligned}
$$

if $\mu$ is a positive odd integer, where $\theta_{n \nu}=2 \pi, \eta_{n \nu}=\eta_{0}$ if $\nu=2^{n}$. We choose a 
sequence $\left\{b_{n}\right\}_{1}^{\infty}$ in $(0,1)$ with $a_{n+1}<b_{n}<a_{n}(n=1,2, \cdots)$. Let (see Fig. 3)

$$
S_{n \mu}=\left\{b_{n}<|z|<a_{n},\left|\arg z-\theta_{n \mu}\right|<\eta_{n \mu}\right\} \quad\left(0 \leqq \mu \leqq 2^{n}-1, n \geqq 1\right) .
$$

Observe that any positive integer $k$ has a unique expression $k=2^{n}+\mu-1$ with positive integer $n$ and nonnegative integer $\mu$ satisfying $\mu \leqq 2^{n}-1$. We set

$$
Y_{k}=S_{n \mu} \quad\left(k=2^{n}+\mu-1\right) .
$$

Then the sequence $\left\{\bar{Y}_{n}\right\}_{1}^{\infty}=\left\{\bar{S}_{n \mu}\right\} \quad\left(0 \leqq \mu \leqq 2^{n}-1, n \geqq 1\right)$ is a $y$-sequence. If we choose the sequence $\left\{b_{n}\right\}$ so as to make the sequence $\left\{b_{n}-a_{n+1}\right\}_{1}^{\infty}$ converges to zero enough rapidly, i.e. satisfying (18) and (19) below then we can show that $\operatorname{dim}\left\{\bar{S}_{n \mu}\right\}=\mathrm{c}$ in the similar way as in nos. 10 and 11.

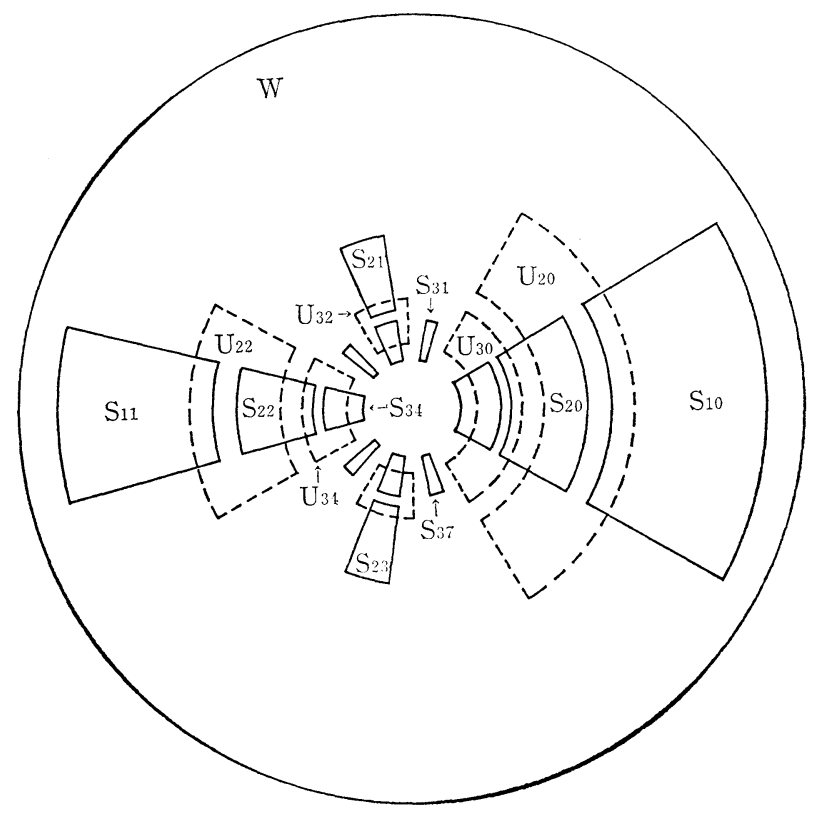

Fig. 3.

Fix a sequence $\left\{\delta_{n}\right\}_{2}^{\infty}$ in $(0,1)$ with $a_{n+1}+\delta_{n+1}<a_{n}-\delta_{n}(n=1,2, \cdots)$, where $\delta_{1}=0$. We set for every integer $n$ with $n \geqq 2$ and every even integer $\mu$ with $0 \leqq \mu \leqq 2^{n}-1$

and (see Fig. 3)

$$
U_{n \mu}=\left\{|| z\left|-a_{n}\right|<\hat{o}_{n},\left|\arg z-\theta_{n \mu}\right|<2 \eta_{n \mu}\right\}
$$

$$
U=\bigcup_{n=2}^{\infty} \bigcup_{\nu=0}^{2 n-1-1} U_{n, 2 \nu}
$$

Then we have $\bar{U}_{n \mu} \cap \bar{U}_{k \nu}=\varnothing((n, \mu) \neq(k, \nu))$. The first property which $\left\{b_{n}\right\}$ has 
to satisfy is

$$
b_{n}<a_{n+1}+\delta_{n+1} \quad(n=1,2, \cdots) .
$$

We consider subregions $W$ and $W_{n}(n=1,2, \cdots)$ of $\Omega$ given by

$$
\begin{gathered}
W=\Omega-\bigcup_{n=1}^{\infty} \bigcup_{\mu=0}^{2 n-1} \bar{S}_{n \mu}, \\
W_{n}=W-\bigcup_{k=1}^{n} \bigcup_{\mu=0}^{2 k-1}\left\{0<|z| \leqq a_{n},\left|\arg z-\theta_{k \mu}\right| \leqq \eta_{k \mu}\right\} \\
-\bigcup_{k=n+1}^{\infty} \bigcup_{\mu=0}^{2 k-1}\left\{0<|z| \leqq a_{k},\left|\arg z-\theta_{k \mu}\right| \leqq \eta_{k \mu}\right\}
\end{gathered}
$$

and denote by $g(\zeta, z), g_{n}(\zeta, z)$ the Green's functions on $W, W_{n}$, respectively. Fix a reference point $a$ in $W-\bar{U}$ of the Martin kernels $k(\zeta, z)=g(\zeta, z) / g(\zeta, a)$, $k_{n}(\zeta, z)=g_{n}(\zeta, z) / g_{n}(\zeta, a)$ on $W, W_{n}$, respectively and let $\delta W_{n}, \delta_{1} W_{n}$ be the Martin boundary of $W_{n}$ over $\delta \Omega: z=0$, the set of minimal points in $\delta W_{n}$, respectively. If we set $\Lambda=\{0,1\}^{\boldsymbol{N}}$ then the cardinal number of $\Lambda$ is $\mathrm{c}$ and every point $x$ in $\Lambda$ defines unique minimal point $p_{x}$ in $\delta_{1} W_{n}=\delta_{1} W_{1}$ : we have $\delta W_{n}=$ $\delta_{1} W_{n}=\delta W_{1}=\delta_{1} W_{1}=\left\{p_{x}: x \in \Lambda\right\}$ and we may choose a family $\left\{\Gamma_{x}: x \in \Lambda\right\}$ of pairwise disjoint curves $\Gamma_{x}$ in $W-\bar{U}$ converging to $\delta \Omega: z=0$ such that

$$
\lim _{\Gamma_{x} \ni z \rightarrow 0} k_{n}\left(p_{y}, z\right)= \begin{cases}+\infty & (x=y) \\ 0 & (x \neq y)\end{cases}
$$

for every $x, y$ in $\Lambda$ and positive integer $n$. Consider the harmonic function $u_{n x}$ on $W_{n-1}$ with boundary values $k_{n}\left(p_{x}, z\right)$ on $\partial W_{n-1}$ for every $x$ in $\Lambda$ and integer $n$ with $n \geqq 2$. Finally we fix a sequence $\left\{\varepsilon_{n}\right\}_{1}^{\infty}$ in $(0,1)$ with $\sum_{1}^{\infty} \varepsilon_{n}<1, \Pi_{1}^{\infty}\left(1-\varepsilon_{n}\right)$ $\geqq 1 / 2$. Then the second property which $\left\{b_{n}\right\}$ has to satisfy is

$$
u_{n+1, x}(z) \leqq \varepsilon_{n}
$$

on $W-U$ for every $x$ in $\Lambda$. We set $v_{1 x}=k_{1}\left(p_{x}, z\right)$ and

$$
v_{n x}(z)=\left(\prod_{k=2}^{n}\left(1-u_{k x}(a)\right)\right)^{-1} k_{n}\left(p_{x}, z\right) \quad(x \in \Lambda, n \geqq 2) .
$$

Assume (19) is valid for every $n$. Then $\left\{v_{n x}\right\}$ converges to a function $v_{x}$ in $H P(W ; \partial W)$ uniformly on every compact subset of $W$ and $v_{x}$ has the same limit as that of $k_{n}\left(p_{x}, z\right)$ at $\delta \Omega: z=0$ along $\Gamma_{y}$ :

$$
\lim _{\Gamma_{y} \ni z \rightarrow 0} v_{x}(z)= \begin{cases}+\infty & (x=y) \\ 0 & (x \neq y)\end{cases}
$$

for every $y$ in $\Lambda$. Then we have $\operatorname{dim}\left\{\bar{S}_{n \mu}\right\} \geqq$ c. Since the dimension of any aj-sequence is at most $c$, we conclude that $\operatorname{dim}\left\{\bar{S}_{n \mu}\right\}=c$. 


\section{REFERENCES}

[1] C. Constantinescu und A. Cornea, Ideale Ränder Riemannsher Flächen, Springer (1963).

[2] M. NAKAI, Densities without Evans solutions, Tôhoku Math. J., 56 (1974), 363-370.

[3] M. NAKAI, Picard principle for finite densities, Nagoya Math. J., 70 (1978), 7-24.

[4] M. NAKaI, The range of Picard dimensions, Proc. Japan Acad., 55 (1979), 379-383.

[5] R. Phelps, Lectures on Choquet's Theorem, Van Nostrand Math. Studies \#7, 1965.

\author{
Mitsuru Nakai \\ Department of Mathematics \\ NAgoya Institute of TECHNOLOGY \\ Gokiso, Showa, Nagoya 466 \\ JAPAN
}

Toshimasa Tada

Department of Mathematics

Daido Institute of TechNology

Daido, Minami, Nagoya 457

JAPAN 